\title{
Methicillin Resistant Staphylococcus aureus in Health Care Workers of a Tertiary Care Infectious Disease Hospital in Nepal
}

\author{
Priyanka Shah ${ }^{1}$, Binod Dhungel ${ }^{1}$, Anup Bastola ${ }^{2}$, Megha Raj Banjara ${ }^{1}$, Komal Raj Rijal ${ }^{1}$, Prakash \\ Ghimire $^{1}$ \\ ${ }^{1}$ Central Department of Microbiology, Tribhuvan University, Kathmandu, Nepal \\ ${ }^{2}$ Sukraraj Tropical and Infectious Disease Hospital, Teku, Kathmandu
}

Corresponding author: Dr. Komal Raj Rijal, Central Department of Microbiology, Tribhuvan University, Kirtipur, Kathmandu, Email: komal.rijal@cdmi.tu.edu.np

\begin{abstract}
Objectives: Acquisition of mecA gene in infectious strains of Methicillin resistant Staphylococcus aureus (MRSA) are considered as one of the potential virulence factors that enables the host bacteria to carry out several nosocomial and community-acquired infections. The main aim of this study was to determine the prevalence of MRSA, their antibiogram and mecA gene in the bacterial isolates obtained from the asymptomatic healthcare workers (HCWs) working in Sukraraj Tropical and Infectious Disease Hospital (STIDH), Kathmandu Nepal.

Methods: This prospective cross-sectional study involved the collection of nasal and hands swab of 125 randomly selected HCWs from December 2019 to February 2020. Conventional microbiological methods were used to isolate and identify S. aureus. Antimicrobial susceptibility testing was done by modified Kirby Bauer disc diffusion method. MRSA was confirmed by using cefoxitin disc. Detection of mecA gene in the chromosome which was extracted by Phenol: Chloroform: isoamyl alcohol DNA extraction method, amplified by using PCR and visualized by running agarose gel electrophoresis.
\end{abstract}

Results: The overall and MRSA carriage rate among the HCWs was found to be $28 \%(35 / 125)$ and $10.4 \%(13 / 125)$ respectively. S. aureus carriage rate was highest among sanitation staffs $(34.2 \%)$ followed by pharmacy staffs (33.3\%), laboratory personnel (18.8\%), doctors (9.1\%) and nurses (7.5\%). Similarly, $34.2 \%(13 / 38)$ of the $S$. aureus isolates were resistant to methicillin, $31.6 \%(12 / 38)$ were inducible-clindamycin resistant and 63.2\% (24/38) of them were multi-drug resistant (MDR). All the 13 MRSA isolates harbored the mecA gene.

Conclusion: Carriage rate of MRSA among HCWs was high and alarming, indicating the prompt need of intervention measures to curb the growth and spread of resistant isolates in the hospital settings. Effective surveillance (of infectious diseases) and establishment of advanced diagnostic facilities can assist in estimating the actual burden of the MRSA which in turn helps to formulate and implement the appropriate policies and infection-control programs to address the increasing antimicrobial resistance in the country.

Keywords: MRSA, Health care workers, Staphylococcus aureus, Nasal carriage

\section{INTRODUCTION}

Staphylococcus aureus-a human commensal and opportunistic pathogen-constitutes the major causative agent of several bacterial infections. Despite the advancement and availability of several antibiotic

Date of Submission: August 16, 2020

Published Online: December, 2020 therapies, staphylococcal infection is still remains as one of the most frequent infections in hospitalized patients causing a wide variety of clinical manifestations ranging in severity from superficial infections such as cutaneous infections to severe invasive diseases like bacteremia (Chakolwa et al. 2019).

Date of Acceptance: October 5, 2020

DOI: https:/ / doi.org/10.3126/tujm.v7i0.33786 
Antibiotics are the miracle drugs in treatment of the infections caused by pathogenic strains of bacteria. However, due to extensive and irrational use of antibiotic has led to the emergence of antimicrobial resistance (AMR), a condition in which bacteria shows the resistance against the antibiotics prescribed against it (Mazzon 2016). Like all other AMR problems, methicillin-resistant S. aureus (MRSA) has also emerged as a major barrier in the management of nosocomial and community-acquired infections. Following the introduction of penicillin in 1940, S. aureus showed resistance against penicillin due to its ability to produce penicillinase, an enzyme that hydrolyses and inactivates the penicillin group of antibiotics. First case of penicillin-resistant $S$. aureus strain was detected in 1942. Methicillin, a semi synthetic penicillin was then developed in the late 1950s to treat penicillin resistant $S$. aureus but the widespread use and misuse of methicillin and other semi-synthetic penicillin led to the emergence of methicillin-resistance, first case reported in 1961 (Barber 1961). Methicillin resistance is chiefly mediated due to the acquisition of a new penicillin binding protein PBP-2' (expressed by an exogenous gene, mecA) which has low affinity to the most $\beta$-lactam antibiotics (Archer 1998). There occurs a wave in the outbreaks associated with MRSA strains which is associated with higher mortality rates, prolonged hospital stays and unwanted economic burden due to the increased cost of treatment (Lakhundi and Zhang 2018). An individual colonized with MRSA has a fourfold increased risk of subsequent infection than by other susceptible strains of S. aureus (Safdar and Bradley 2008).

MRSA once confined to hospitals, health care environments and patients frequenting such facilities, now has become a serious problem for communities due to its emergence as a major cause of the communityassociated infections (Khatri et al. 2017; Lakhundi and Zhang 2018). Since mid-1990s, sudden increase in the number of MRSA infections reported in the communities has been associated with the recognition of new MRSA strains. Such novel strains are often called as community-associated MRSA (CA-MRSA) strains. These strains which principally used to cause skin and soft tissue infections are now responsible for hospital care-associated infection as well (David and Daum 2010).

HCWs serve as the link between hospitals and the communities, which plays a significant role in cross-contamination of nosocomial and community acquired infections (El Aila et al. 2017). Asymptomatic colonization of MRSA among HCWs is a prerequisite for subsequent endogenous infection and dissemination of the strains to the hospital environment (Abimana et al. 2019). Early identification of MRSA carriers among HCWS may help to minimize the potential outbreaks in several hospitals.

Drug-resistant, often multidrug-resistant strains of the commensal and opportunistic bacteria including MRSA, methicillin resistant S. epidermidis (MRSE), vancomycin resistant Staphylococcus aureus (VRSA) and some strains of Pseudomonas spp, Haemophilus spp, Streptococcus spp are the major problem in the infection control strategies. Aside from socioeconomic burden and augmented risk of dissemination, these resistant strains may go undetected in the resource poor settings of Low- to lower-middle income countries (LMICs) which results the emergence of novel strains furthering the challenges in the fight with AMR (Thapa et al. 2020). A number of studies are conducted in Nepal which have estimated the prevalence of MRSA ranging from $26.1 \%$ to $57.1 \%$ (Ansari et al. 2014; Kumari et al. 2008; Rijal et al. 2008; Khanal and Jha 2010; Raut et al. 2017; Bhomi et al. 2016; Rijal et al. 2008; Shahi et al. 2018; Thapa et al. 2020; Kandel et al. 2020). Although there seems a large number of studies are based on MRSA but almost all studies are merely relied on the phenotypic detection. Therefore, there is still a paucity of researches to estimate the molecular detection and characterization of the resistant genes. This study explores the prevalence of MRSA strains, their antibiogram and the molecular detection of mecA gene in order to corroborate the need and importance of molecular detection techniques and their reliability in the precise detection of resistant strains.

\section{MATERIALS AND METHODS}

\section{Study design, study site and sample population}

This prospective cross-sectional study was conducted for a period of three months from December 2019 to February 2020. Sample collection and processing was conducted at Sukraraj Tropical and Infectious Disease Hospital (STIDH), Teku while the molecular detection of the mecA gene was carried out at Central Department of Microbiology, Tribhuvan University, Kathmandu. All purposively selected HCWs above 18 years of age from the hospital who consented to provide sociodemographic information along with nasal and hand 
swab samples, were included in the study. Similarly, HCWs under 18 years of age, who were unable provide written consent for socio-demographic information were excluded from the study. A total of 250 ( $n=125$ nasal; $n=125$ hand) swab samples were obtained from $125 \mathrm{HCWs}$, each of the study participant provided a nasal swab and a hand swab.

\section{Collection and transport of samples}

Swab samples were collected aseptically by using sterile cotton swabs pre-moistened with sterile normal saline, following standard methodology (Cheesbrough 2012). Briefly, the swab was rotated inside the anterior nares of each nostril for 2-3 times with slight finger pressure on the outside of the nose in order to assure good contact between swab and the chamber of the nostril. Without contaminating the swab, the procedure was repeated for the second nostril and the swabs were transported to the laboratory with the labels of subject's identification number and other required information (Cheesbrough 2012). No transport medium was used as the microbiology department and sample collection sites were adjacent to each other. Drying of swab was prevented by keeping the swab in tubes containing nutrient broth.

\section{Isolation and identification of S. aureus}

Swab samples were inoculated into mannitol salt agar (MSA) and blood agar (BA) and were incubated at $37^{\circ} \mathrm{C}$ for $24 \mathrm{hrs}$. S. aureus was identified on the basis of colony characteristics, Gram's staining, and biochemical assays such as catalase, oxidase and coagulase test (Cheesbrough 2012).

\section{Antibiotic susceptibility testing and screening of MDR S. aureus}

Antimicrobial susceptibility test (AST) was performed in-vitro using modified Kirby-Bauer disc diffusion method on Mueller-Hinton Agar (MHA) as per CLSI guidelines (2019). Following antibiotic discs (Hi Media Laboratories, Pvt. Limited, India) were

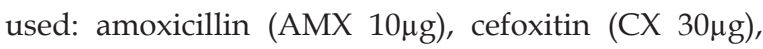

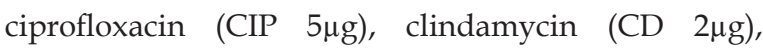

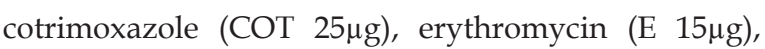
gentamicin $(\mathrm{GEN} 10 \mu \mathrm{g})$, penicillin $(\mathrm{P} 10 \mu \mathrm{g})$, tetracycline $(\mathrm{TE} 30 \mu \mathrm{g})$, cloxacillin (COX 10 $\mu \mathrm{g}$ ) and ceftriaxone (CTR $30 \mu \mathrm{g})$. Results were interpreted on the basis of CLSI guidelines (2019). Isolates showing non-susceptibility (either resistant or intermediate) to at least one agent in three or more antimicrobial categories were identified as MDR (Magiorakos et al. 2011). Confirmed S. aureus isolates were preserved by using $20 \%$ glycerol in TSB for further analysis.

\section{Screening of MRSA}

Methicillin resistance was detected by using cefoxitin disk of $30 \mu \mathrm{g}$ on MHA plates by Kirby-Bauer disc diffusion method and was interpreted according to CLSI guidelines (2019). Isolates showing zone of inhibition of $\leq 21 \mathrm{~mm}$ around cefoxitin disc (30 $\mu \mathrm{g})$ were considered as MRSA (CLSI 2019).

Detection of inducible-clindamycin resistance (ICR) Inducible-clindamycin resistant was detected by double disk approximation test (D-test) as per CLSI (2019) guidelines. In this test, a 0.5 McFarland's standard suspension of $S$. aureus was prepared and plated onto MHA. An erythromycin disk $(15 \mu \mathrm{g})$ and clindamycin disk $(2 \mu \mathrm{g})$ were placed $15 \mathrm{~mm}$ apart edgeto-edge on MHA plate. Plates were analyzed after 18 hours of incubation at $37^{\circ} \mathrm{C}$. Isolates were considered inducible-clindamycin resistant when an isolate was resistant to erythromycin but sensitive to clindamycin showing flattening of the zone of inhibition of $\geq 21 \mathrm{~mm}$ around clindamycin producing a " $D$ " shaped blunting towards erythromycin disk ( $D$-test positive).

\section{Extraction of DNA}

All phenotypically confirmed MRSA isolates were treated under Phenol: chloroform: isoamyl alcohol extraction method for the detection of mecA gene. In this method, isolates were grown in Luria Bertani (LB) broth at $37^{\circ} \mathrm{C}$ in an orbital shaker at 120 rpm for 24 hours. $1.5 \mathrm{ml}$ of liquid culture was transferred to microfuge tube of $1.5 \mathrm{ml}$ volume. Then, the bacterial cells were lysed with $3-5 \mathrm{mg} / \mathrm{ml}$ lysozyme in the presence of $1 / 10$ volume of $10 \%$ Sodium Dodecyl Sulfate (SDS) at high $\mathrm{PH}^{\mathrm{H}}$ and the lysate was then neutralized. Subsequent deproteinization with 1:1 Phenol: Chloroform was done and then genomic DNA was precipitated with ethanol by spinning at high speed (Shrestha and Adhikari 2014).

\section{PCR amplification of mecA gene}

Thus extracted mecA gene was further amplified by polymerase chain reaction (PCR). In PCR test, the crude lysates were used as a DNA template whereas (mecA PF1)5'- ACT GCT ATC CAC CCT CAA AC$3^{\prime}$ and (mecA PR1) 5'- CTG GTG AAG TTG TAA TCTGG-3' were used as forward and reverse primer respectively (Vatansever et al. 2016). A final $10 \mu \mathrm{l}$ 
solution was prepared by mixing up of $5 \mu$ l master mix, $1 \mu \mathrm{l}$ each of forward and reverse primer, $1 \mu \mathrm{l}$ DNA, and $2 \mu$ nuclease free water. The amplification cycle consisted of initial denaturation at $95^{\circ} \mathrm{C}$ for 120 seconds, denaturation at $95^{\circ} \mathrm{C}$ for 30 seconds, annealing at $56.2^{\circ} \mathrm{C}$ for 30 seconds, extention at $72^{\circ} \mathrm{C}$ for 20 seconds, and 29 cycles of amplification at $72^{\circ} \mathrm{C}$ for 5 minutes. The amplified products were then subjected to gel electrophoresis using $1.5 \%$ agarose gel stained with ethidium bromide.The final product (163-bp DNA fragment) was then visualized under UV light. The presence of the gene was confirmed by comparing with a positive control using 100 bp DNA ladder (Molecular Biology, Thermo Fisher Scientific Company) in the gel run. The band of 163-bp was considered positive for the mecA gene (Vatansever et al. 2016; Oliveira and de Lencastre 2011).

\section{Statistical analysis}

All the data were analyzed by using IBM SPSS statistics
23.0 version software. Frequency and percentage for descriptive and Chi Square test with cross tab for inferential statistics were used. A p-value of $<0.05$ was considered as statistically significant.

\section{RESULTS}

\section{Distribution of bacterial growth in swab samples}

Among the 250 swabs processed, 241 (96.4\%) samples showed the growth of bacteria in which $81.2 \%(203 / 250)$ of them were coagulase-negative Staphylococci (CONS) while 15.2\% (38/250) showed growth of $S$. aureus (Figure 1). Out of 38 positive samples, $42.1 \%$ $(n=16)$ were isolated from nasal swab while $57.9 \%$ $(n=22)$ were isolated from hands swab. About $10.4 \%$ $(13 / 125)$ of the HCWs had colonization with S. aureus only, $15.2 \%(19 / 125)$ of them had hands colonization alone while $2.4 \%(3 / 125)$ of them had both nasal and hands colonization. However, $72 \%$ of the staff's both nasal and hand swabs were free from $S$. aureus.

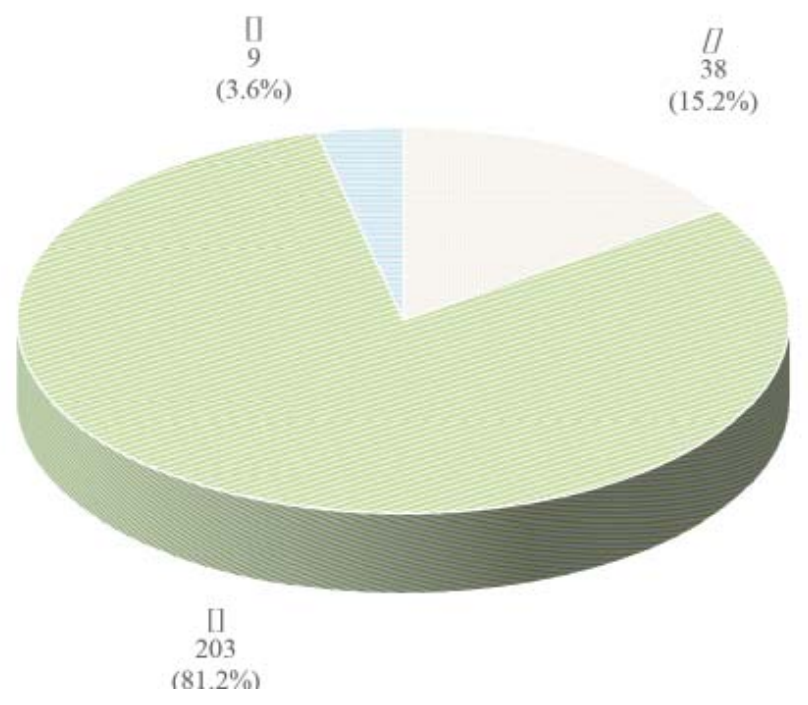

Figure 1: Distribution of bacterial growth

\section{Antibiotic resistance of $S$. aureus}

Regarding antimicrobial susceptibility pattern of all 38 S. aureus isolates, a high proportion of $S$. aureus isolates (73.7\%) showed resistance towards amoxycillin. Also, $68.4 \%, 60.5 \%, 47.3 \%, 36.8 \%, 34.2 \%, 15.8 \%$,
$12 \%, 7.9 \%$ and $7.9 \%$ of the isolates were resistant to erythromycin, cloxacillin, ciprofloxacin, ceftriaxone, cefoxitin, gentamicin, clindamycin, cotrimoxazole and tetracycline respectively (Figure 2). 


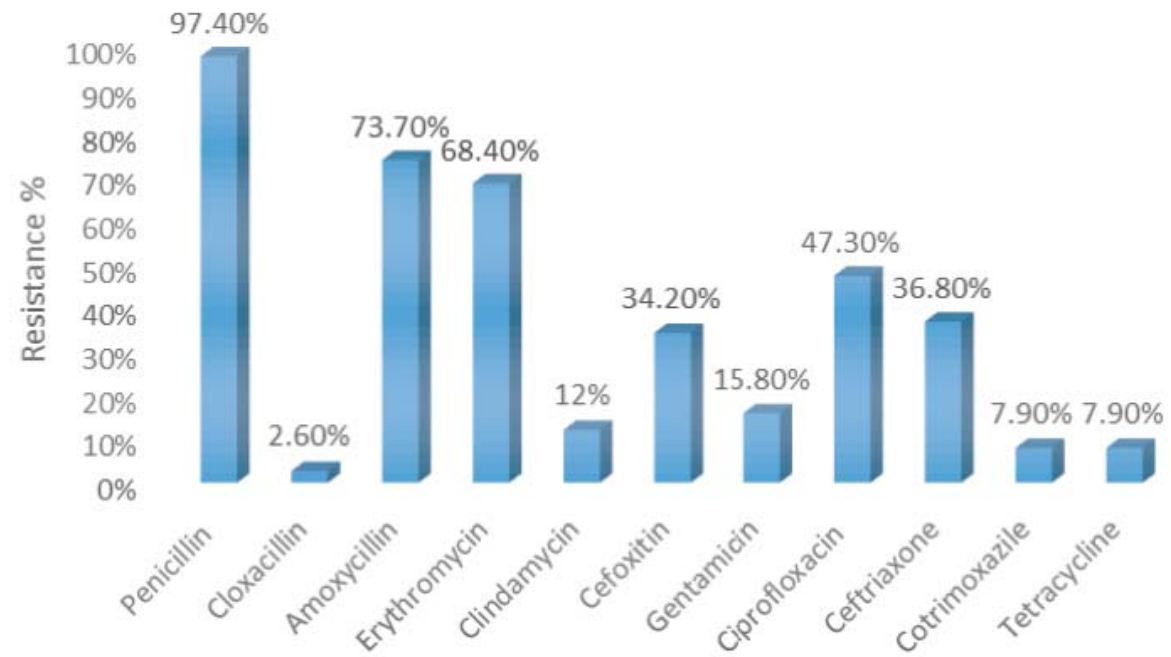

Figure 2: Antibiotic resistance pattern of $S$. aureus isolates

\section{Phenotypic screening of MRSA}

Out of 38 S. aureus isolates, $34.2 \%$ (13) were resistant to methicillin. Among which, 61.5\% (8/13) isolates were obtained from nasal swab whereas 38.5\% (5/13) were obtained from hand swab (Figure 3 ).

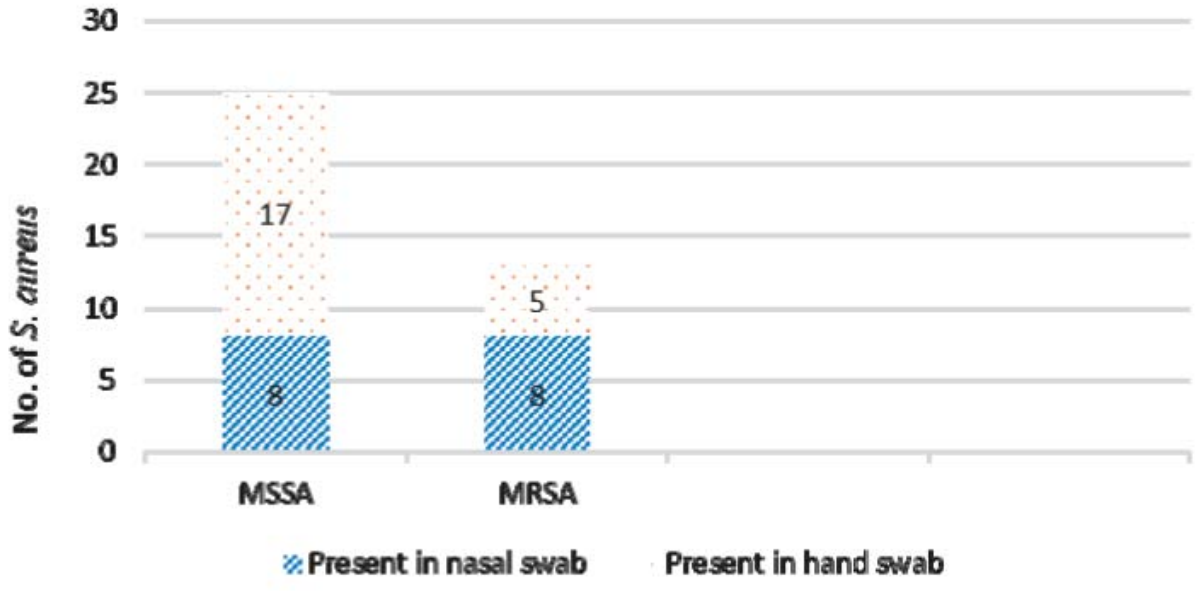

Figure 3: MSSA and MRSA among S. aureus isolates

Distribution of S. aureus and MRSA according to age and gender of the subjects

Among the total studied participants, 24 were males and 101 were females. Respectively 32.7\% (33/101) and $20.8 \%(5 / 24)$ female and male participants were found to have harbored S. aureus. Similarly, 10.9\% (11/101) and $8.35 \%(2 / 24)$ of the male and female subjects respectively harbored MRSA strains.
This study included HCWs of the age between 16-80 years old. Colonization with MRSA was highest (6.3\%; $2 / 32$ ) among the age group $>50$ years, followed by the age group of $26-50$ years $(5.6 \% ; 8 / 144)$ and $15-25$ years $(4.1 \%$; $3 / 74)$. However, there was no significant association between the bacterial load (MRSA) and, gender and age groups $(\mathrm{p}=0.67)$ (Table 1$)$. 
Table 1: Age and gender wise distribution of S. aureus and MRSA

\begin{tabular}{|c|c|c|c|c|c|c|}
\hline \multirow{4}{*}{$\begin{array}{c}\text { Age of health care } \\
\text { workers }\end{array}$} & \multirow{4}{*}{$\begin{array}{c}\text { Total no. of samples } \\
\text { N }\end{array}$} & \multicolumn{4}{|c|}{ Gender of the health care workers } & \multirow{4}{*}{$\begin{array}{c}\text { Total MRSA } \\
\text { N (\%) }\end{array}$} \\
\hline & & \multicolumn{2}{|c|}{ Male $(n=24)$} & \multicolumn{2}{|c|}{ Female $(n=101)$} & \\
\hline & & S. aureus & MRSA & S. aureus & MRSA & \\
\hline & & N (\%) & N (\%) & N (\%) & N (\%) & \\
\hline $18-25$ & 74 & 0 & 0 & $6(8.1)$ & $3(4.1)$ & $3(4.1 \%)$ \\
\hline $26-50$ & 144 & $3(2.03)$ & $1(0.7)$ & $23(16)$ & $7(4.9)$ & $8(5.6 \%)$ \\
\hline Above 50 & 32 & $2(6.3)$ & $1(3.1)$ & $4(12.5)$ & $1(3.1)$ & $2(6.3 \%)$ \\
\hline Total & 250 & 5 & 2 & 33 & 11 & $13(5.2 \%)$ \\
\hline
\end{tabular}

S. aureus and MRSA carriage among different groups of health profession

Among the total staffs, the highest percentage of sanitation staffs $(34.2 \% ; 13 / 38)$ were colonized with S. aureus followed by pharmacy staffs $(33.3 \% ; 2 / 6)$ and lab personnel (18.8\%; 6/32). However, MRSA carriage was observed highest in pharmacy staffs $(16.7 \% ; 1 / 6)$ followed by lab personnel $(12.5 \% ; 4 / 32)$, sanitation staffs $(7.9 \% ; 3 / 38)$ and nurses $(5 \% ; 4 / 80)$. This distribution of the bacterial load and MRSA were not found to be statistically associated with the various professions (wards) of the HCWs ( $\mathrm{p}=0.152)$ (Table 2).

Table 2: Distribution of S. aureus and MRSA carriage among different groups of health professions

\begin{tabular}{lccc}
\hline Profession of health care workers & $\begin{array}{c}\text { Total no. of samples } \\
\text { N }\end{array}$ & $\begin{array}{c}\text { No. of S. aureus } \\
\text { N (\%) }\end{array}$ & $\begin{array}{c}\text { MRSA } \\
\text { N (\%) }\end{array}$ \\
\hline Doctor & 22 & $2(9.1 \%)$ & 0 \\
Nurse & 80 & $6(7.5 \%)$ & $4(5 \%)$ \\
Lab Personnel & 32 & $6(18.8 \%)$ & $4(12.5 \%)$ \\
Pharmacist & 6 & $2(33.3 \%)$ & $1(16.7 \%)$ \\
Health Assistant & 18 & $3(16.7 \%)$ & 0 \\
Sanitation Staff & 38 & $13(34.2 \%)$ & $3(7.9 \%)$ \\
Interns & 30 & $3(10 \%)$ & 0 \\
Others & 24 & $3(12.5 \%)$ & $1(4.2 \%)$ \\
\hline
\end{tabular}

Distribution of isolates in different departments of the hospital

In this study, the rate of $S$. aureus carriage was highest among pharmacy staffs $(33.3 \% ; 2 / 6)$ followed by laboratory staffs $(20.3 \% ; 13 / 64)$ and ICU staffs $(16.7$ $\% ; 1 / 6)$ and staffs from the emergency department $(16.7 \% ; 3 / 18)$. However, MRSA colonization was higher in HCWs from pharmacy $(16.7 \% ; 1 / 6)$ and ICU $(16.7 \% ; 1 / 6)$ followed by HCWs from ward staffs $(6.9 \% ; 5 / 72)$ and lab staffs $(6.3 \% ; 4 / 64)$ respectively. This distribution was not statistically significant with p-value of 0.628 , which suggests that MRSA carriage rate and departments of duty of HCWs are independent of each other (Table 3).

Table 3: Distribution of $S$. aureus and MRSA isolates in different departments

$\left.\begin{array}{lccc}\hline \multicolumn{1}{c}{\text { Department of duty }} & \text { Total no. of samples } & \text { No. of S. aureus } & \text { MRSA } \\ \text { N (\%) } & \text { N (\%) }\end{array}\right)$


Distribution of MRSA and MSSA on the basis of duration of employment

Highest number of $S$. aureus was seen in those HCWs who served the hospital for less than 10 years as $52.6 \%$ $(20 / 38)$ of the S. aureus and 35\% (7/20) of the MRSA isolates were recovered from them. This was followed by the service year of 20-30 years with $23.7 \%(9 / 38)$
S. aureus load and $22.2 \%(2 / 9)$ of MRSA. Those who served for 20-30 years showed the rate of $23.7 \%(9 / 38)$ of $S$. aureus while the rate was $22.2 \%$ (2/9) of MRSA. It was then followed by 10-20 years and 30-40 years of employment with $21.1 \%(8 / 38)$ and $2.6 \%(1 / 38)$ of $S$. aureus carriage respectively. However, the distribution was statistically insignificant as $(\mathrm{p}=0.532)$ (Figure 4).

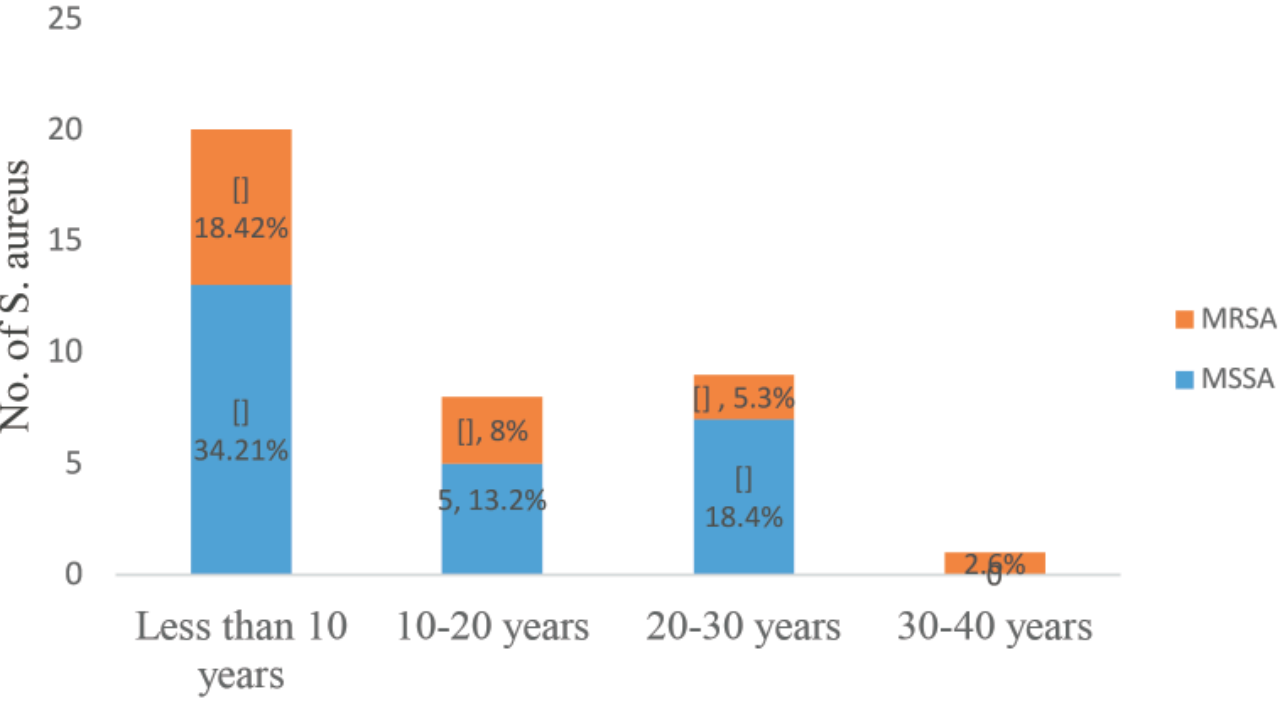

Figure 4: MSSA and MRSA distribution according to years of employment

Antibiotic susceptibility pattern of MSSA and MRSA

All (13) of the MRSA isolates were resistant towards penicillin, amoxycillin, cefoxitin and ceftriaxone. 12 $(92.3 \%)$ isolates were resistant to erythromycin. $92.3 \%$ $(12 / 13)$ of the total MRSA isolates were susceptible to cloxacillin, clindamycin and gentamicin. Almost equal portion $(84.6 \% ; 11 / 13)$ of the MRSA isolates were susceptible to both Cotrimoxazole and Tetracycline.
However, $60 \%(15 / 25)$ of MSSA isolates were resistant to amoxycillin followed by $56 \%$ (14/25) for erythromycin. All of the MSSA isolates were susceptible towards cefoxitin and cloxacillin. 96\% (24/25) of MSSA were susceptible for ceftriaxone, cotrimoxazole and tetracycline. $92 \%(23 / 25)$ and $80 \%(20 / 25)$ of them were susceptible towards clindamycin and gentamicin respectively (Table 4).

Table 4: Antibiotic susceptibility patten of MSSA and MRSA

\begin{tabular}{lcccc}
\hline \multirow{2}{*}{ Antibiotic disc } & \multicolumn{2}{c}{ MSSA (N=25), $\mathbf{n}(\%)$} & \multicolumn{2}{c}{ MRSA (N=13), $\mathbf{n}(\%)$} \\
\cline { 2 - 5 } & $\begin{array}{c}\text { Sensitive (S) } \\
\mathbf{N}(\%)\end{array}$ & $\begin{array}{c}\text { Resistant (R) } \\
\mathbf{N}(\%)\end{array}$ & $\begin{array}{c}\text { Sensitive (S) } \\
\mathbf{N}(\%)\end{array}$ & $\begin{array}{c}\text { Resistant (R) } \\
\mathbf{N}(\%)\end{array}$ \\
\hline Penicillin & $1(4 \%)$ & $24(96 \%)$ & 0 & $13(100 \%)$ \\
Cloxacillin & $25(100 \%)$ & 0 & $12(92.3 \%)$ & $1(7.7 \%)$ \\
Amoxycillin & $10(40 \%)$ & $15(60 \%)$ & 0 & $13(100 \%)$ \\
Erythromycin & $11(44 \%)$ & $14(56 \%)$ & $1(7.7 \%)$ & $12(92.3 \%)$ \\
Clindamycin & $23(92 \%)$ & $2(8 \%)$ & $12(92.3 \%)$ & $1(7.4 \%)$ \\
Cefoxitin & $25(100 \%)$ & 0 & 0 & $13(100 \%)$ \\
Gentamicin & $20(80 \%)$ & $5(20 \%)$ & $12(92.3 \%)$ & $1(7.7 \%)$ \\
Ciprofloxacin & $14(56 \%)$ & $11(44 \%)$ & $6(46.2 \%)$ & $7(53.8 \%)$ \\
Ceftriaxone & $24(96 \%)$ & $1(4 \%)$ & 0 & $13(100 \%)$ \\
Cotrimoxazole & $24(96 \%)$ & $1(4 \%)$ & $11(84.6 \%)$ & $2(15.4 \%)$ \\
Tetracycline & $24(96 \%)$ & $1(4 \%)$ & $11(84.6 \%)$ & $2(15.4 \%)$ \\
\hline
\end{tabular}


Prevalence of multi-drug resistant and inducible -clindamycin resistant $S$. aureus isolates

Among 38 S. aureus isolates, 24 (63.2\%) of them were MDR. Similarly, all of the MRSA isolates were MDR.
Out of 38 S. aureus isolates, $12(31.5 \%)$ isolates were screened as inducible-clindamycin resistance. Among 12 isolates, 7 were MRSA (Table 5).

Table 5: Prevalence of multi-drug resistant and inducible -clindamycin resistant S. aureus

\begin{tabular}{ccccccc}
\hline $\begin{array}{c}\text { No. of S. } \\
\text { aureus }\end{array}$ & MRSA N (\%) & MDR N (\%) & $\begin{array}{c}\text { MDR isolates } \\
\text { excluding } \\
\text { MRSA, N (\%) }\end{array}$ & $\begin{array}{c}\text { D-test Positive } \\
\text { N (\%) }\end{array}$ & $\begin{array}{c}\text { MRSA with } \\
\text { Positive D-test, } \\
\text { N (\%) }\end{array}$ & $\begin{array}{c}\text { MSSA with } \\
\text { Positive } \\
\text { D-test, N (\%) }\end{array}$ \\
\hline 38 & $13(34.2 \%)$ & $24(63.2 \%)$ & $11(29 \%)$ & $12(31.6 \%)$ & $7(18.4 \%)$ & $5(13.2 \%)$ \\
\hline
\end{tabular}

Prevalence of mecA gene among MRSA isolates

All of the 13 MRSA isolates were confirmed to have harbored

mecA gene (Table 6). The mecA gene was detected under gel electrophoresis with product size $163 \mathrm{bp}$ (Figure 5).

Table 6: Detection of mecA gene in MRSA

\begin{tabular}{lcc}
\hline Sample & $\begin{array}{c}\text { No. of MRSA isolates } \\
\text { N }\end{array}$ & $\begin{array}{c}\text { No. of mec A positive MRSA } \\
\text { N (\%) }\end{array}$ \\
\hline Nasal swab & 8 & $8(100 \%)$ \\
Hand swab & 5 & $5(100 \%)$ \\
\hline
\end{tabular}

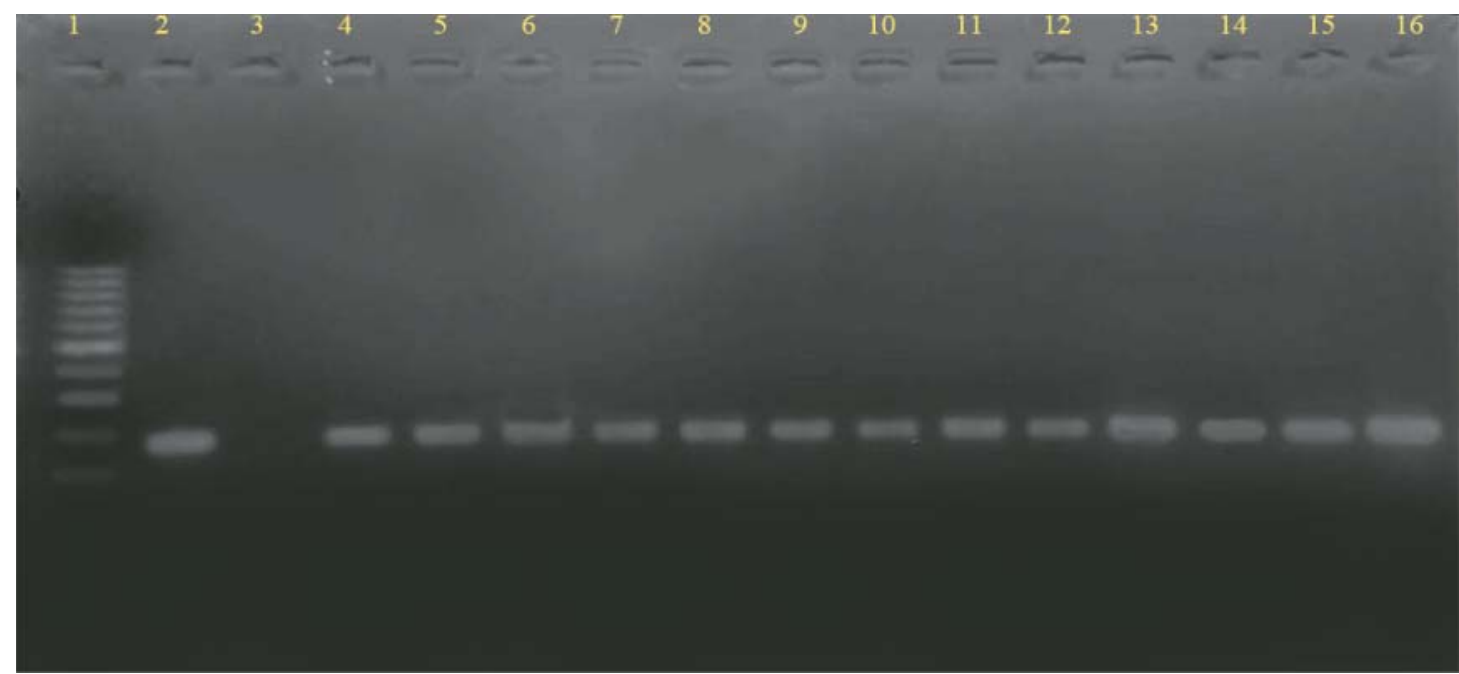

Figure 5: Confirmation of mecA gene by gel documentation of PCR products. Lane 1: 100bp DNA ladder, Lane 2: Positive control, Lane 3: Negative control, Lane 4-16: isolates with positively amplified mecA gene (163bp).

\section{DISCUSSION}

Healthcare workers (HCWs) are one of the major reservoirs of $S$. aureus as they often serve as the interface between hospitals and communities. Therefore, HCWs are required to screened for carriage of pathogenic and resistant strains of $S$. aureus so that potential nosocomial and community acquired infections and can be prevented and the chain of transmission can break at the earliest. As MRSA has emerged as a serious public health problem, screening of HCWs allows appropriate management of the colonized staff members. In this study, nearly one fifth of the HCWs were colonized with the commensal, $S$. aureus. Of the positive individuals, one-third (34.2\%) of the isolated bacteria were the strains of MRSA - all of those isolates harbored the mecA gene.

Some previous studies have reported the prevalence of S. aureus and that of MRSA among the HCWs as $23.7 \%$ and $4.6 \%$ respectively (Albrich and Harbarth 2008; Khatri et al. 2017). In this study, the overall prevalence was slightly higher than the average rate which may be due to negligence on infection control guidelines, safety and sanitation measures among them. Moreover, nasal carriage rate of S. aureus and MRSA among the HCWs 
was found to be $12.8 \%(16 / 125)$ and $6.4 \%(8 / 125)$ respectively. Similarly, the carriage rates of $S$. aureus and MRSA in the hands of HCWs were $17.6 \%(22 / 125)$ and $4 \%(5 / 125)$ respectively. The findings of our study are in line with the previous study by Khatri et al. (2017), in which the nasal carriage rates of S. aureus and MRSA were $18.3 \%$ and $7.5 \%$ respectively. Also, $72.7 \%$ of $S$. aureus colonization was reported by Lama et al. (2017), $15.7 \%$ by Khanal et al. (2015), 20.37\% by Sah et al. (2013), 25\% by Shakya et al. (2010). The nasal carriage rate of $S$. aureus in this study was lower than the studies conducted elsewhere in Nepal. Prevalence of $S$. aureus in this study when compared internationally also shows variation. A study conducted by Shibabaw et al. (2013) from Northeast Ethiopia and Abimana et al. (2019) from Central Uganda reported the rate of $28.8 \%$ from each of the studies. Lower prevalence than our study was reported from Kenya with $18.3 \%$; Zambia (17.1\%), Kuwait (21\%) and India (21.4\%) (Omuse et al. 2012). Higher prevalence was from Iran (31\%), Gaza (31\%), Germany (33.8\%), Chile (34.9\%), Libya (39\%), Central Uganda (41.9\%), Tanzania (41.4\%) and Nigeria (64\%) (Shibabaw et al. 2013; Abimana et al. 2019; El Aila et al. 2017). Similarly, nasal MRSA carriage rate was also lower than the findings by Shakya et al. (2010) with $10 \%$ and Khatri et al. (2017) with $7.5 \%$ but was higher than those reported by Shrestha et al. (2009) with 2.3\% and Khanal et al. (2015) with 3.4\% from Nepal. MRSA prevalence in present study is higher than some overseas findings from Iran (5.3\%) and Zambia (5.7\%) but lower than Ethiopia (12.7\%), Libya (19\%), Egypt (13.5\%), and Gaza (25.5\%) (Chakolwa et al. 2019). These differences in the prevalence of $S$. aureus and its strains between countries and hospitals are probably due to differences in the quality and size of samples, variation in sampling techniques, microbiological procedures, different interpretation guidelines, local infection control standards and the local prevalence of MRSA. Moreover, different levels of commitment while performing laboratory works by the investigator also contribute to these differences (El Aila et al. 2017, Chakolwa et al. 2019).

Socio-demographic characteristics including age, gender, profession, length of healthcare services, and services in different departments within the same hospital have been reported to influence the carriage of S. aureus (Kandel et al. 2020). Also in this study, significant differences in the prevalence of $S$. aureus among HCWs have been well documented with the variation in such characteristics listed in the aforementioned sentence.

In the antimicrobial susceptibility assay, most of the isolates were susceptible to carbapenems and gentamicin. Higher susceptibility of $S$. aureus isolates towards these antibiotics was also reported in previous studies (Thapa et al. 2020; Kandel et al. 2020; Sah et al. 2013) and augmented resistance to gentamicin was also reported in another studies (Thulunga et al. 2015). Similarly, AST of MRSA isolates showed resistance to most of the antibiotics used except carbapenems, vancomycin and gentamicin. Our findings resonate well with some of other findings reported earlier (Thapa et al. 2020; Kandel et al. 2020; Sah et al. 2013; Belbase et al. 2017; Rijal et al. 2008). However, in another study, ciprofloxacin was effective against MRSA isolates (Shrestha 2013).

Furthermore, this study revealed nearly one-third (31\%) of $S$. aureus isolates as inducible-clindamycin resistance which could be easily misidentified as clindamycin susceptible in Kirby-Bauer disk diffusion method. Therefore, D-test should be routinely performed to all the $S$. aureus isolates in clinical microbiology laboratory to guide clinicians for appropriate use of clindamycin. The prevalence of inducible-clindamycin resistance among $S$. aureus observed in this study is higher than the prevalence reported by many other researches done in Nepal as well as done internationally. From Nepal, Adhikari et al. (2017) reported $11.48 \%, 12.4 \%$ by Ansari et al. (2016), 12.1\% by Sah et al. (2013), 3\% by Mishra et al. (2013), 28.7\% by Kumari et al. (2008). Varying prevalence rates of inducible-clindamycin resistance have been reported from worldwide.

mecA gene responsible for conferring the drug-resistant to the MRSA and MDR isolates of $S$. aureus. Detection of mecA gene serves as an evidence of the presence of MRSA among entire $S$. aureus isolates. This statement has been further reinforced by various findings from Sudan (Maimona et al. 2014), Saudi Arabia (Meshref and Omer, 2011), Iraq (Al-Zu'bi et al. 2004), Japan (Hotta et al. 1999), India (Mehndiratta et al. 2009), Australia (Cloney et al. 1999) and USA (Murakami et al. 1991). In comparison to those studies, burden of mecA was similar in our study as all of the MRSA isolates were tested positive for the gene. This could be due to the increased awareness, augmented infection controls 
in the health facility under the study. However, other intrinsic factors also need to be assessed in future studies which might have been competing and inhibiting the expression of mecA in MRSA isolates in large number of study findings (Kandel et al. 2020). Globally, a number of studies have documented the absence of mecA in MRSA isolates (Aziz et al. 2014).

A study from Nigeria reported the absence of 5 major SCCmec types, gene products of PBP2 and mecA genes in phenotypically confirmed MRSA isolates. This finding informs the existing of other intrinsic factors such as the probability of hyper-production of $\beta$-lactams, responsible for conferring the resistance (Olayinka et al. 2009). There can also be the possibility of the alterations in various amino acids in protein binding proteins cascade (PBPs 1, 2 and 3). Such alterations have been reported to cause 3 amino acid substitutions (with identical or different amino acids) in all variants of PBP (Ba X et al. 2014). Hence, the existence of several other intrinsic factors aside for mecA suggests that detection of the gene alone cannot assure the detection of resistance. This important point needs to be pondered by the regional and reference laboratories while formulating and implementing the policy. Moreover, a more strict policy should be considered to discourage the over-thecounter (OTC) use of drugs and irrational prescriptions and use of antibiotics among HCWs and patients. Furthermore, HCWs, irrespective of the absence of clinical complaints are advised to be routinely screened for the potential carriage and transfer of the pathogenic strains of the bacteria.

\section{CONCLUSION}

More than one-fifth of the HCWs were colonized with $S$. aureus while one in ten isolates were MRSA and all of the MRSA tested positive for mecA gene in this study. The findings in this study reinforce the need for more commitment towards infection control measures that meet the standard protocols and aims at reducing the spread of infection by MRSA among susceptible individuals. Augmentation of diagnostic facilities along with antimicrobial stewardships can be recommended to combat the burgeoning spread of resistant bacteria.

\section{ACKNOWLEDGEMENTS}

The authors acknowledge the concerned persons at Sukraraj Tropical and Infectious Disease Hospital (STIDH) and Central Department of Microbiology, Tribhuvan University, Kirtipur, Kathmandu, Nepal for their contributions.

\section{CONFLICT OF INTEREST}

The authors declare that they have no competing interests.

\section{REFERENCES}

Abimana JB, Kato CD and Bazira J (2019) MethicillinResistant Staphylococcus aureus Nasal Colonization among Healthcare Workers at Kampala International University Teaching Hospital, Southwestern Uganda. Can J Infect Dis Med Microbiol 2019:1-7.

Albrich WC and Harbarth S (2008) Health-care workers: source, vector, or victim of MRSA? Lancet Infect Dis 8(5):289-301.

Al-Zu'bi E, Bdour S, Shehabi AA (2004) Antibiotic resistance patterns of mecA-positive Staphylococcus aureus isolate from clinical specimens and nasal carriage. Microb Drug Resist 10:321-324.

Ansari S, Gautam R, Shrestha S, Ansari SR, Subedi SN and Chhetri MR (2016) Risk factors assessment for nasal colonization of Staphylococcus aureus and its methicillin resistant strains among pre-clinical medical students of Nepal. BMC Res Notes 9(1):1-8.

Archer GL (1998) Staphylococcus aureus : A Well-Armed Pathogen. Clin Infect Dis 26:1179-81.

Aziz HW, Al-Dulaimi TH, Al-Marzoqi AH, Ahmed NK (2014) Phenotypic detection of resistance in Staphylococcus aureus isolates: detection of (mecA and fem $\mathrm{A})$ gene in methicillin resistant Staphylococcus aureus (MRSA) by polymerase chain reaction. J Nat Sci Res 4:112-118.

Baber, M (1961) Methicillin-resistant staphylococci. J Clin Pathol 14:385-393.

Ba X, Harrison EM, Edwards GF, Holden MT, Larsen AR, Petersen A, Skov RL, Peacock SJ, Parkhill J, Paterson GK, Holmes MA (2014) Novel mutations in penicillin-binding protein genes in clinical Staphylococcus aureus isolates that are methicillin resistant on susceptibility testing, but lack the mec gene. J Antimicrob Chemother (3):594-7.

Belbase A, Pant ND, Nepal K, Neupane B, Baidhya R, Baidya R, Lekhak B (2017) Antibiotic resistance and biofilm production among the strains of Staphylococcus aureus isolated from pus/wound swab samples in a tertiary care hospital in Nepal. 
Ann Clin Microbiol Antimicrob 16(1):15.

Bhomi U, Rijal KR, Neupane B, Shrestha S, Chaudhary M, Acharya D, Thapa Shrestha U, Adhikari N, Ghimire P (2016) Status of inducible clindamycin resistance among macrolide resistant Staphylococcus aureus. Afr J Microbiol Res 10:280-284.

Chakolwa G, Samutela MT, Kwenda G, Mulundu G, Mwansa J, Hang'ombe BM, Simulundu E and Lukwesa C (2019) Carriage rate and antimicrobial resistance profiles of Staphylococcus aureus among healthcare workers at a large tertiary referral hospital in Lusaka, Zambia. Sci African 5:1-9.

Cheesbrough M (2012) District Laboratory Practice in Tropical Countries. Second Edition Update. Part 2. Cambridge University Press, 116-123.

Cloney L, Marlowe C, Wong A, Chow R, Bryan R (1999) Rapid detection of mecA in methicillin resistant Staphylococcus aureus using cycling probe technology. Mol Cell Probes 13:191-197.

CLSI (2019) Performance standards for antimicrobial susceptibility testing. CLSI Document M100. Clinical and Laboratory Standards Institute; $29^{\text {th }}$ edition.

David MZ and Daum RS (2010) Community-associated methicillin-resistant Staphylococcus aureus: Epidemiology and clinical consequences of an emerging epidemic. Clin Microbiol Rev 23(3):616-687.

El Aila NA, Al Laham NA and Ayesh BM (2017) Nasal carriage of methicillin resistant Staphylococcus aureus among health care workers at Al Shifa hospital in Gaza Strip. BMC Infect Dis 17(1):1-7.

Hotta K, Ishikawa J, Ishii R, Saitoii F, Kira K, Arakawa Y, Ike Y (1999) Necessity and usefulness of detection by PCR of mecA and $a a c\left(6^{\prime}\right)-I e+a p h\left(2^{\prime \prime}\right)$ genes for identification of arbekacin resistant MRSA. Jpn J Antibiot 52:525-532.

Kandel SN, Adhikari N, Dhungel B, Thapa Shrestha U, Angbuhang, KB, Karki G, Adhikari B, Banjara, MR, Rijal KR, and Ghimire P (2020) Characteristics of Staphylococcus aureus isolated from clinical specimens in a Tertiary Care Hospital, Kathmandu, Nepal. Microbiology Insights 13: 1-6.

Khanal LK, Jha BK (2010) Prevalence of methicillin resistant Staphylococcus aureus (MRSA) among skin infection cases at a hospital in Chitwan, Nepal. Nepal Med Coll J 12:224-228.

Khanal R, Sah P, Lamichhane P, Lamsal A, Upadhaya S and Pahwa VK (2015) Nasal carriage of methicillin resistant Staphylococcus aureus among health care workers at a tertiary care hospital in Western Nepal. Antimicrob Resist Infect Control 4(1):3-7.

Khatri S, Pant ND, Bhandari R, Shrestha KL, Shrestha CD, Adhikari N and Poudel P (2017) Nasal Carriage Rate of Methicillin Resistant Staphylococcus aureus among Health Care Workers at a Tertiary Care Hospital in Kathmandu, Nepal. J Nepal Health Res Counc 15(35):26-30.

Kumari N, Mohapatra TM, Singh YI (2008) Prevalence of methicillin-resistant Staphylococcus aureus (MRSA) in a tertiary-care hospital in Eastern Nepal. J Nepal Med Assoc 47:53-56.

Lakhundi S and Zhang K (2018) Methicillin-resistant Staphylococcus aureus:Molecular characterization, evolution, and epidemiology. Clin Microbiol Rev 31(4):1-103.

Lama U, Shah D and Shrestha UT (2017) Vancomycin Resistant Staphylococcus aureus Reported from Tertiary Care Hospital in Nepal. TUJM 4(1): 6372.

Magiorakos A, Srinivasan A, Carey RB, Carmeli Y, Falagas ME, Giske CG, Harbarth $S$ and Hindler JF (2011) Bacteria: an International Expert Proposal for Interim Standard Definitions for Acquired Resistance. Clin Microbiol Infect 18:268-281.

Maimona A, Eliman E, Suhair R, Miskelyemen AE, Mogahid ME (2014) Emergence of vancomycin resistant and methicillin resistant Staphylococcus aureus in patients with different clinical manifestations in Khartoum State. J Amer Sci 10:106-110.

Mazzon D (2016) [Ethical use of antibiotics in the era of multi resistance: a common good for the individual or the society?]. Recent Prog Med. 107(2):71-4.

Mehndiratta PL, Bhalla P, Ahmed A, Sharma YD (2009) Molecular typing of methicillin- resistant Staphylococcus aureus strains by PCR-RFLP of SPA gene: a reference laboratory perspective. Indian J Med Microbiol 27:116-122. 
Meshref AA, Omer MK (2011) Detection of (mecA) gene in methicillin resistant Staphylococcus aureus (MRSA) at Prince A/Rhman Sidery. J Med Genet Genomic 3:41-45.

Murakami K, Minamide W, Wada K, Nakamura E, Teraoka H, Watanabe S (1991) Identification of methicillin-resistant strains of staphylococci by polymerase chain reaction. J Clin Microbiol 29:2240-2244.

Olayinka BO, Olayinka AT, Obajuluwa AF, Onaolapo JA, Olurinola PF (2009) Absence of mecA gene in methicillin-resistant Staphylococcus aureus isolates. Afr J Infect Dis 3:49-56.

Oliveira DC, de Lencastre H (2011) Methicillinresistance in Staphylococcus aureus is not affected by the overexpression in trans of the mecA gene repressor: a surprising observation. PLoS One 6:e23287.

Omuse G, Kariuki S and Revathi G (2012) Unexpected absence of methicillin-resistant Staphylococcus aureus nasal carriage by healthcare workers in a tertiary hospital in Kenya. Journal of Hospital Infection 80(1):71-73.

Rijal KR, Pahari N, Shrestha BK, Nepal AK, Paudel B, Mahato P, Skalko-Basnet N (2008) Prevalence of methicillin resistant Staphylococcus aureus in school children of Pokhara. Nepal Med Coll J 10:192-195.

Rijal KR, Shrestha N, Pahari N, Shrestha B, Paudel B, Nepal A, Ghimire P, Rijal B (2008) Methicillin resistance Staphylococcus aureus in patients visiting western regional hospital, Pokhara. JIOM 30:21-25.

Raut S, Bajracharya K, Adhikari J, Pant SS, Adhikari B (2017) Prevalence of methicillin resistant Staphylococcus aureus in Lumbini Medical College and Teaching Hospital, Palpa, Western Nepal. BMC Res Notes 10:187.

Safdar N and Bradley EA (2008) The Risk of Infection after Nasal Colonization with Staphylococcus aureus. Am J Med 121(4):310-315.

Shahi K, Rijal KR, Adhikari N, Thapa Shrestha U, Banjara MR, Sharma VK, Ghimire P (2018)
Methicillin resistant Staphylococcus aureus: prevalence and antibiogram in various clinical specimens at Alka Hospital. TUJM 5:77-82.

Sah P, Rijal KR, Shakya B, Tiwari BR and Ghimire P (2013) Nasal Carriage Rate of Staphylococcus aureus in Hospital Personnel of National Medical College and Teaching Hospital and their Antibiotic Susceptibility Pattern. JHAS 3(1):21-3.

Shakya B, Shrestha S and Mitra T (2010) Nasal carriage rate of methicillin-resistant Staphylococcus aureus among at National Medical College Teaching Hospital, Birgunj, Nepal. Nepal Med Coll J 12(1): 26-29.

Shibabaw A, Abebe T and Mihret A (2013) Nasal carriage rate of methicillin resistant Staphylococcus aureus among Dessie Referral Hospital Health Care Workers; Dessie, Northeast Ethiopia. Antimicrob Resist Infect Control 2: 25.

Shrestha B, Pokhrel BM and Mohapatra TM (2009) Staphylococcus aureus Nasal Carriage among Health Care Workers in a Nepal Hospital. Brazilian J Infect Dis 13(5):322.

Shrestha B (2013) Comparative prevalence of MRSA in two Nepalese tertiary care hospitals. Open J Clin Diag 3:67.

Shrestha UT and Adhikari N (2014) A practical manual for microbial genetics. Max Printing Press 1:86130.

Thapa S, Adhikari N, Dhungel B, Thapa M, Shrestha UT, Banjara MR, Rijal KR and Ghimire P (2020) Etiology of ocular infections and minimum inhibitory concentration of multidrug- resistant Staphylococcus aureus isolates to vancomycin, ciprofloxacin and chloramphenicol. Acta Scientific Microbiology 3(6): 134-145.

Thulunga J, Bhattarai A, Basnyat S, Singh SK, Singh A, Rijal KR (2015) Screening of Staphylococcus aureus as nasal carrier from hospital personnel. International Journal of Advances In Case Reports 2(8):493-497.

Vatansever L, Sezer C, Bilge N (2016) Carriage rate and methicillin resistance of Staphylococcus aureus in food handlers in Kars City, Turkey. Springerplus 5:608. 